\title{
ANALYTICITY WITHOUT ANALYSIS
}

\author{
WILLIAM C. FOX
}

The purpose of this note is to point out an easy but striking consequence (Theorem 1) of a theorem proved by Walter Rudin in 1953, [1].

THEOREM 1. Let $X$ be a topological manifold of dimension two, and let $f$ and $g$ be functions sending $X$ into the Riemann sphere. There exists a conformal structure for $X$ relative to which both $f$ and $g$ are meromorphic and nonconstant if and only if every member of the complex algebra generated by $f$ and $g$ is continuous and interior and at least one member is also light, excepting constants.

This "consequence" is striking because it enables one to define the various conformal structures carried by $X$ using functions defined only on all of $X$ and properties only of a topological or algebraic sort. Differentiability is never mentioned.

Since the algebra in question contains a member, $h$, which is light and interior, one concludes by Stoilow's theorem that $h$ is locally topologically equivalent with power functions, i.e., $h$ is a ramified cover of the sphere. Thus there exists a unique conformal structure for $X$ relative to which $h$ is meromorphic. For proof, see, e.g., [2, pp. 119-120]. If, relative to this structure, $c$ is an analytic homeomorphism sending a disk into $X$, then the algebra generated by $h(c), f(c)$, and $g(c)$ consists entirely of interior mappings (i.e., of mappings which send open sets onto open sets) and has one member, $h(c)$, which is known to be meromorphic. If these functions have no poles, then by Rudin's theorem (see Theorem 2 below) they are all analytic. Consequently, $f$ and $g$ are analytic (except for poles) on $X$ relative to the same conformal structure. Riemann's theorem guarantees then that they are meromorphic on $X$ relative to that structure. This completes the proof, for the converse is standard.

THEOREM 2 (Rudin). Let a be a complex algebra of functions continuous on the open unit disk. Suppose there exists a basis, B, for that disk's topology, consisting entirely of Jordan domains such that $f \mid B$ attains its maximum modulas on the boundary of $B$ whenever $f \in a$ and

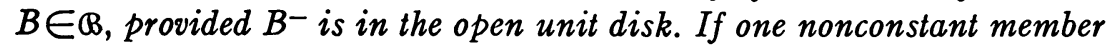
of $Q$ is analytic, then every member is analytic.

Evidently, Theorem 2 may be applied whenever the relation

Received by the editors February 10, 1961 and, in revised form, March 3, 1961. 


$$
\partial f(U) \subset f(\partial U)
$$

holds for every $f \in Q$ and, e.g., every conditionally compact set $U$; such functions are said by Whyburn [3, p. 112] to be quasi-open. Since a quasi-open and light map is necessarily interior, it follows that one may replace "interior" in Theorem 1 with "quasi-open." It is important to note how much weaker than quasi-openness are $\mathrm{Ru}$ din's hypotheses in Theorem 2 so far as individual functions are concerned. For example, Rudin's hypotheses (applied to a single function) permit folding whereas quasi-openness does not, as one sees easily upon consideration of the map $f$ defined by the rules

$$
f(z)=\left\{\begin{array}{ccc}
z & \text { when } & |z| \leqq 1, \\
\frac{z}{|z|^{2}} & \text { when } & |z| \geqq 1 .
\end{array}\right.
$$

Indeed, Whyburn [4] has shown recently that those mappings whose restrictions to multiply connected conditionally compact Jordan domains either have zero minimum modulus (computed relative to an arbitrary point as origin) or else attain their minimum modulus on the boundary are exactly the quasi-open mappings. Thus Rudin's extreme modulus property (for a single function) is required to hold only relative to the origin whereas Whyburn's must hold relative to every point serving as the origin.

\section{BIBLIOGRAPHY}

1. Walter Rudin, Analyticity, and the maximum modulus principle, Duke Math. J. 20 (1953), 449-451.

2. Lars V. Ahlfors and Leo Sario, Riemann surfaces, Princeton Univ. Press, Princeton, N. J., 1960.

3. Gordon T. Whyburn, Topological analysis, Princeton Univ. Press, Princeton, N. J., 1958.

4. - Quasi-open mappings, Rev. Math. Pures Appl. 2 (1957), 47-52.

Tulane University 